CKD without diabetes who were enrolled in the Modification of Diet in Renal Disease study. The cohort comprised mainly white and relatively young individuals (mean age $49.9 \pm 12.9$ years) who had a low prevalence of cardiovascular disease and an over-representation of polycystic kidney disease as the cause of CKD. Although the incidence of mortality increased with age, stage of CKD (i.e. with decreasing glomerular filtration rate), and level of proteinuria, the rate of ESRD was higher than that of death in every subgroup of glomerular filtration rate examined. Overall, the rate of ESRD (median follow-up 88 months) was almost four times that of death (median follow-up of 123 months; 84 events per 1,000 person-years vs 23 events per 1,000 person-years). Only in patients over 65 years of age was the rate of death comparable to that of ESRD, although ESRD remained prevalent.

The authors conclude that in relatively healthy, young patients with CKD without diabetes, treatment should focus on prevention of cardiovascular events and delaying progression of kidney disease.

Original article Menon V et al. (2008) Long-term outcomes in nondiabetic chronic kidney disease. Kidney Int 73: 1310-1315

\section{Potential kidney donors accept higher donor risk than do clinicians or recipients}

Young et al. have quantified acceptance of health risk to kidney donors among potential donors, potential recipients, and transplant professionals. Their analysis found that potential donors are most willing to accept risk to their long-term health, whereas potential recipients are most averse to donor health risks.

This observational Canadian study included 111 potential kidney recipients who had stage 4 or 5 chronic kidney disease or were receiving dialysis, 112 potential donors who were relatives or friends of the potential recipients and had no obvious contraindication to kidney donation, and 51 transplant professionalsincluding nephrologists, surgeons, living-donor coordinators, social workers and a psychologist. Each participant completed a questionnaire to establish the risk thresholds for long-term hypertension, kidney failure and cardiovascular disease in the donor that would stop the participant from proceeding with living-doner kidney donation.

Potential donors were more willing to accept a higher than average risk of donor hypertension, cardiovascular disease or kidney failure than were transplant recipients or transplant professionals; in fact, approximately half of all potential donors were willing to accept risks over three times higher than the estimated risks in a healthy population. A total of $61 \%$ of potential donors agreed that donation was acceptable even if the long-term donor health risks were uncertain, but only $11 \%$ of potential recipients and $32 \%$ of transplant professionals agreed $(P<0.0001)$.

Transplant clinicians should be aware that willingness to accept possible health risks to potential kidney donors varies considerably between themselves, potential donors and potential recipients.

Original article Young A et al. (2008) Differences in tolerance for health risk to the living donor among potential donors, recipients, and transplant professionals. Kidney Int 73: $1159-1166$

\section{No need to screen kidney donors or recipients for Toxoplasma where seroprevalence is low}

Toxoplasmosis is a serious complication of solid-organ transplantation. The highest risk of infection and disease occurs in seronegative individuals who receive a heart transplant from a seropositive donor (donor-recipient mismatch); $50-75 \%$ of these recipients develop symptomatic toxoplasmosis in the absence of prophylaxis. Infection can present as myocarditis, brain abscess or disseminated disease. Little is known about the risk of toxoplasmosis associated with other solid-organ transplantations.

Gourishankar et al. performed a retrospective cohort study of 1,006 adults who underwent solid-organ transplantation at the University of Alberta Hospital in Edmonton, Canada, from 1984 to 1997. Toxoplasma gondii screening results before and at least 3 months after transplantation were available for 907 donor-recipient pairs (including 516 kidney or kidney-pancreas transplantations). The prevalence of $T$. gondii seropositivity was relatively low $(13.4 \%$ in donors and $17.8 \%$ in recipients), as expected in a North American setting. Among the 51 kidney or kidney-pancreas donor-recipient 